\title{
A Comparison of the Effect of Sonar Grid Map Building Based on DSmT and DST
}

\author{
Xinde Li, Xinhan Huang, Min Wang and Gang Peng \\ Intelligent Control and Robotics Laboratory, \\ Department of Control Science and Engineering, \\ Huazhong University of Science and Technology, Wuhan 430074,China \\ Tel: 027-87556242;Email:xd1825@163.com
}

\begin{abstract}
Information acquired in map building presents characteristics of uncertainty, imprecision and even high conflict, especially in the course of building grid map using sonar. Here a new method of information fusion-DSmT (Dezert-Smarandache Theory) recently developed from DST and Bayesian theory was introduced to apply to managing and dealing with this uncertainty. Aiming to static environment, classic DSm mathematic modal was established by constructing the general basic belief assignment function, so that the global evidence grid map is built by applying the classic DSm rule. Pioneer II virtual mobile robot served as an experiment platform. By comparing the numbers of acquiring-information spot, the quality of grid map building and the time in need of finishing the global map building, better fusion results than that with DST are gotten. Especially when dealing with high conflictive information from sonar grid map building, DSmT was more effective and successful than DST. In short, this study not only found a new method for building map under static environment, but also supplied with a theory foundation for us to further apply Hybrid DSmT to dynamic unknown environment and multi-robots' building map together.

Index Terms - DSmT, Information fusion, Grid map building,
\end{abstract} DST, Uncertainty.

\section{INTRODUCTION}

The study on exploration of entirely unknown environment for intelligent mobile robots has being a popular and difficult subject for experts in robots' field for a long time. Because of the contradiction between self-localization and map building, some one compares it as a puzzle chickens and eggs $[1,2]$. To solve this puzzle, some experts in robot's field have proposed many methods in self-localization and map building respectively, for example, Markov[3] or Monte carlo[4] self-localization, grid map[5,6], geometrical feature or topological map, etc. Of course, here we apply DSmT (DezertSmarandache Theory) and DST to sonar grid map building, which is one of the most successful expression methods in building map. Due to sonar sensors' restriction of physical characteristics, information acquired in grid map building presents characteristics of uncertainty, imprecision and even high conflict. DST proposed by Dempster and Shafer since 1976 [7] has been widely applied since Professor Smets proposed a model of TBM (Transfer Belief Model)[8], which offered an explanation on it. But it is very difficult to deal with highly conflictive information, because its conflict factor isn't equal to one. Aiming to this difficulty, A new method of information fusion for DSmT proposed by Jean Dezert

\footnotetext{
* Supported by National Natural Science Foundation of China (\#69585003) to Xinhan Huang
}

(French) and Florentin Smarandache (American) based on Bayesian theory and DS theory come forth since 2003, which is a general, flexible and valid arithmetic of fusion $[9,10]$.

\section{SIMPLE REVIEW OF DSmT}

\section{A. The Frame of Discernment for DSmT}

Let $\Theta=\left\{\theta_{1}, \theta_{2}, \cdots, \theta_{n}\right\}$, here $\Theta$ is the frame of discernment, which includes $\mathrm{n}$ finite focal elements $\theta_{i}(i=1, \cdots n)$. Because the focal element is not precisely defined and separated, so that no refinement of $\Theta$ in a new larger set $\Theta_{\text {ref }}$ of disjoint elementary hypotheses is possible.

\section{B. Hyper-power of DSmT}

The hyper-power set $D^{\Theta}$ is defined as the set of all compositions built from elements of $\Theta$ with $U$ and $\cap$ (generates $D^{\Theta}$ under operators $\bigcup$ and $\cap$ ) operators such that

$$
\begin{aligned}
& \text { 1) } \phi, \theta_{1}, \theta_{2}, \theta_{3} \cdots \theta_{n} \in D^{\Theta} \\
& \text { 2) If } A, B \in D^{\Theta} \text {, then } A \cap B \in D^{\Theta} \text { and } A \cup B \in D^{\Theta} \\
& \text { 3) No other elements belong to } D^{\Theta} \text {, except those } \\
& \text { obtained by using rules 1) or 2). }
\end{aligned}
$$

\section{General Belief Function and Plausibility Function}

Let $\Theta=\left\{\theta_{1}, \theta_{2}, \cdots, \theta_{n}\right\}$ is the general frame of discernment. For every evidential source $S$, let us define a set of map of $m(\cdot): D^{\Theta} \in[0,1]$ associated to it (abandoning Shafer's model) by assuming here that the fuzzy/ vague/ relative nature of elements $\theta_{i}(i=1,2,3 \cdots n)$ can be nonexclusive, as well as no refinement of $\Theta$ into a new finer exclusive frame of discernment $\Theta_{r e f}$ is possible. The mapping $m(\cdot)$ is called a generalized basic belief assignment function (gbbaf), if it satisfies

$$
m(\phi)=0 \quad \text { and } \quad \sum_{A \in D^{\Theta}} m(A)=1
$$

The general belief function and plausibility function are defined respectively in almost the same manner as within the DST, i.e.

$$
\begin{aligned}
& \operatorname{bel}(A)=\sum_{B \in D^{\Theta}, B \subseteq A} m(B) \\
& \operatorname{Pl}(A)=\sum_{B \cap A \neq \phi, B \in D^{\Theta}} m(B)
\end{aligned}
$$

\section{Classical (free) DSmT Rule of Combination}


Let $M^{f}(\Theta)$ is a free model of DSmT, and then the classical (free) DSmT rule of combination for $k \geq 2$ sources is given as follows:

$$
\forall A \neq \phi \in D^{\Theta},=\sum_{\substack{X_{1}, \cdots X_{K} \in D^{\Theta} \\\left(X_{1} \cap \cdots X_{K}\right)=A}} \prod_{i=1}^{m_{M^{f}(\Theta)}} m_{i}(A) \cong\left[m_{i} \oplus \cdots m_{k}\right](A)
$$

\section{MODELING FOR SONAR GRID INFORMATION}

Sonar sensors' working principle (shown as Fig 1) is: producing sheaves of cone-shaped wave, and detecting the objects by receiving the reflected wave. Due to the restriction of sonar physical characteristic, metrical data behaves out uncertainty [11]

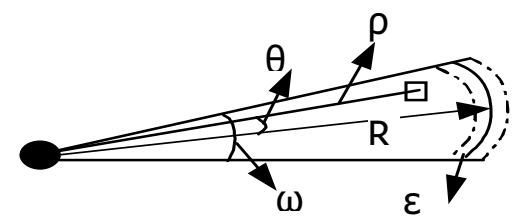

Fig.1: Sketch of the principle of sonar

\section{A. Modeling Based on DSmT}

Pointing to the characteristics of sonar's measurement, here we suppose there are two focal elements in system, that is, $\Theta=\left\{\theta_{1}, \theta_{2}\right\}$, here $\theta_{1}$ means grid is empty, $\theta_{2}$ means occupied, and then we can get its hyper-power set $D^{\Theta}=\left\{\phi, \theta_{1} \cap \theta_{2}, \theta_{1}, \theta_{2}, \theta_{1} \cup \theta_{2}\right\}$. Every grid in environment is scanned $k \geq 2$ times, each of which is viewed as source of evidence. Then we may define a set of map aiming to every source of evidence and construct the general basic belief assignment functions (gbbaf) as follows: $m\left(\theta_{1}\right)$ is defined as the gbbaf for grid-unoccupied (empty); $m\left(\theta_{2}\right)$ is defined as the gbbaf for grid-occupied; $m\left(\theta_{1} \cap \theta_{2}\right)$ is defined as the gbbaf for holding grid-unoccupied and occupied simultaneous (conflict). $m\left(\theta_{1} \cup \theta_{2}\right)$ is defined as the gbbaf for gridignorance due to the restriction of knowledge and experience presently (here referring to the gbbaf for these grids still not scanned presently), it reflects the degree of ignorance of gridunoccupied or occupied.

The gbbaf of a set of map $m(\cdot): D^{\Theta} \rightarrow[0,1]$ is constructed by authors such as the formulae (4) (7) according to sonar physical characteristics.

$m\left(\theta_{1}\right)=E(\rho) \cdot E(\theta)=$

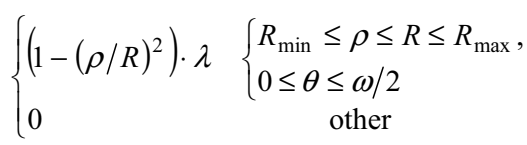

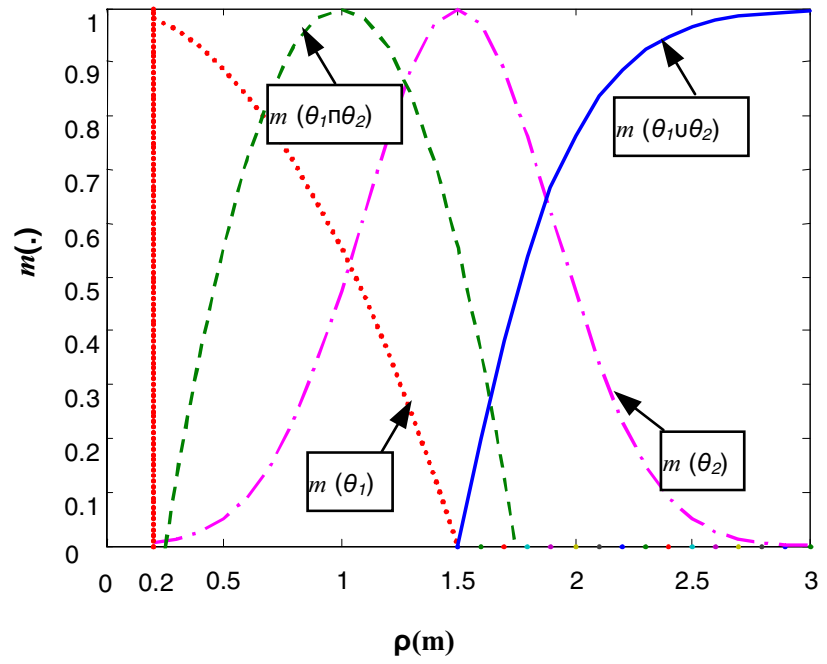

$m\left(\theta_{2}\right)=\mathrm{O}(\rho) \cdot \mathrm{O}(\theta)=$

Fig.2: $m($.$) as function of given by Eq.(4 7)$

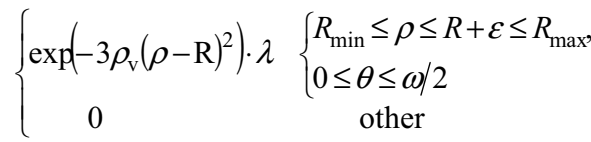

$m\left(\theta_{1} \cap \theta_{2}\right)=$

$\left\{\begin{array}{cl}\left(1-(2(\rho-(R-2 \varepsilon)) / R)^{2}\right) \cdot \lambda & \left\{\begin{array}{l}R_{\min } \leq \rho \leq R \leq R_{\max } \\ 0 \leq \theta \leq \omega / 2\end{array}\right. \\ 0 & \text { other }\end{array}\right.$

$m\left(\theta_{1} \cup \theta_{2}\right)=$

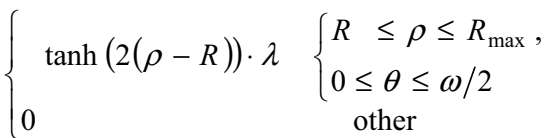

where $\lambda=E(\theta)=O(\theta)$ is given by(see ref.[5] for justification).

$\lambda=E(\theta)=O(\theta)=\left\{\begin{array}{cc}1-(2 \theta / \omega)^{2} & 0 \leq|\theta| \leq \omega \mid 2 \\ 0 & \text { other }\end{array}\right.$

Seen from Fig.2, gbbaf reflects really out the characteristics of sonar information with the shift of in the course of building grid map. Here we assume the range of sonar sensor from $0.2 \mathrm{~m} \sim 3 \mathrm{~m}$. High conflictive information happens about at the point of intersection of two curves between $m\left(\boldsymbol{\theta}_{1}\right)$ and $m\left(\boldsymbol{\theta}_{2}\right)$. The maximum $m\left(\boldsymbol{\theta}_{2}\right)$ happens at $\mathrm{R}$, while the maximum of $m\left(\theta_{1}\right)$ happens at $\mathrm{R}_{\min }=0.2 \mathrm{~m}$. Of course, in order to satisfy the definition of DSmT, and assure the sum of all mass of $m(\cdot)$ to be one, we must renormalize them while acquiring sonar grip information.

\section{B. Modeling Based on DST}

For DST, it isn't a new theory, so here we needn't recall it, and then use it directly. According to the requirement of sonar grid map building, here we also suppose two focal elements in system, that is, $\Theta=\left\{\theta_{1}, \theta_{2}\right\}$, here $\theta_{1}$ means grid is empty, $\theta_{2}$ means occupied, and then we can get its power 
set $2^{\Theta}=\left\{\phi, \theta_{1}, \theta_{2}, \theta_{1} \cup \theta_{2}\right\}$. According to DST, let $m_{D S T}(\phi)=0$,

\section{FRONT}

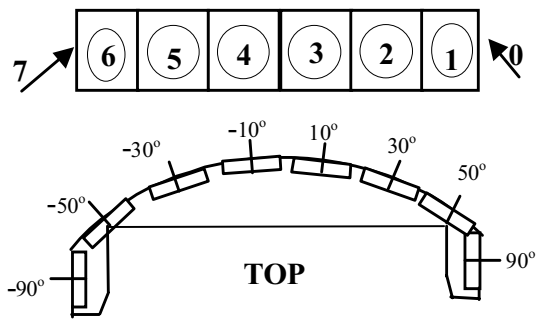

Fig .3: Sketch of the layout of sonars

here $m_{D S T}\left(\theta_{1}\right)$ is defined as the basic belief assignment Function (bbaf) for grid-empty, $m_{D S T}\left(\theta_{2}\right)$ is defined as the basic belief assignment function (bbaf) for grid-occupied, $m_{D S T}\left(\theta_{1} \cup \theta_{2}\right)$ is defined as the basic belief assignment function for grid-ignorance. We may also construct basic belief assignment function such as $m_{D S T}\left(\theta_{1}\right)=m\left(\theta_{1}\right)$, $m_{D S T}\left(\theta_{2}\right)=m\left(\theta_{2}\right) \quad, \quad m_{D S T}\left(\theta_{1} \cup \theta_{2}\right)=m\left(\theta_{1} \cup \theta_{2}\right)$. bbaf reflects still the characteristics of uncertainty for sonar grip map building in Fig.2. Though here we define the same bbaf as DSmT, because the definition of DST must be satisfied, we must renormalize them while acquiring sonar grip information.

\section{EXPERIMENTAL RESULTS}

\section{A. Fusion of Sonar Information}

Pioneer II virtual mobile robot has 16 Sonar sensors. Seen from Fig.3, there are just 8 front sonar sensors shown, their distribution is asymmetrical. Here we only give the procedure of fusion based on DSmT, because of the restriction of paper length. In fact, DST is similar to DSmT. Fusion steps are listed as follow:

We develop the experimental platform with the Visual $\mathrm{c}++$ 6.0, through the protocol of TCP / IP.

1) At the beginning of procedure, we proposed that all grids are fully occupied, that is, $\operatorname{Bel}\left(\theta_{2}\right)=1$, and mobile robot begins from origin of coordinate shown as Fig 4. Therefore, we may order the mobile robot to some spots. Of course, mobile robot' path planning and avoiding obstacles must be considered, but it is not an important point here. Robot may get the information of all sonar sensors at spot $(\mathrm{i}, \mathrm{j})$. For simplified calculation, we apply the arithmetic of restrict spreading, which only computes the grid information in the fan-shaped area that each sonar can scan (shown as Fig 2)[11].

2) Utilizing the model of uncertainty belief established in the third part, through(4) (8), gbbaf (such as $m\left(\theta_{1}\right)$ 、 $m\left(\theta_{2}\right) 、 m\left(\theta_{1} \cap \theta_{2}\right)$ and $m\left(\theta_{1} \cup \theta_{2}\right)$ is computed respectively. If sum of mass is not one, and then we should renormalize it.

3) Judge whether the information of every grid scanned by all sonar sensors is new or not. If yes, and then goto step 1. Otherwise, goto next step.
4) Judge whether the grid is scanned repeatedly or not. If No, save the information of this grid. If Yes, goto step5.

5) Go on judging whether the fusion times is more than two times or not. If yes, and then stop fusing it. Otherwise, goto step 6.

6) Go on fusing it and at the same time, judge further. Whether the grid's information is fused for the first time or not. If it is not the first time, and then compute and update the $\mathrm{Bel}$ at last. And then goto step 7. if it is the first time, goto step 8.

7) Check whether $\mathrm{Bel}$ of all grids to be updated or not. If Yes, and goto step 9. Otherwise, goto step 1 .

8) Update the grid's original mass with the new mass after fusion. Then goto step 1 .

9) Stop and Exit the procedure at last.

\section{B. Comparison of Fusion Result between DSmT and DST}

we suppose the environment (size: $5 \mathrm{~m} \times 5 \mathrm{~m}$ ) partitioned 2500 discrete even rectangular grids $(50 \times 50)$. Objects in rectangular grid map are sketched in Fig 4. Mobile robot wanders there, and gets some points of location for acquiring information. Because the environment is smaller, moreover robot runs less time, the precision for self-localization of

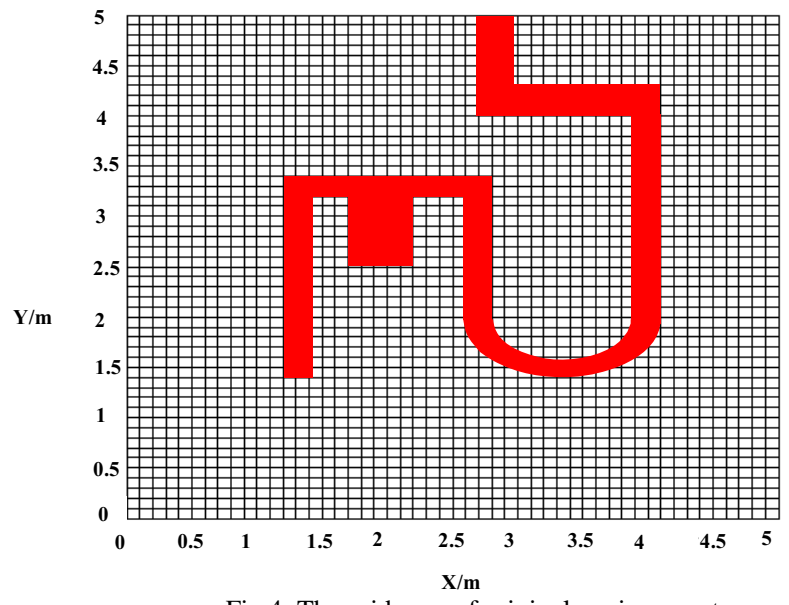

Fig.4: The grid map of original environment

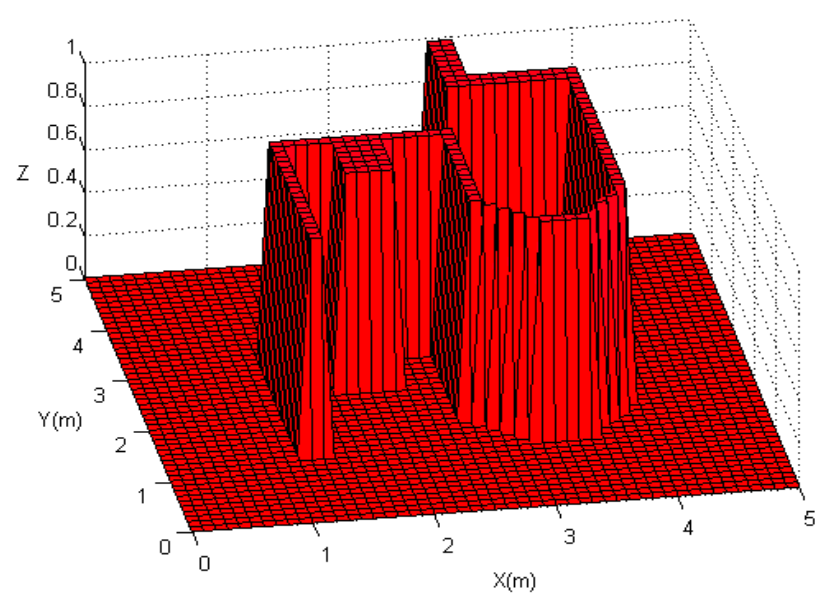

Fig .5: Estimation of grid map with DSmT(here $\mathrm{Z}$ axis represents $\operatorname{Bel}\left(\theta_{2}\right)$ ) 
mobile robot is enough to realize the arithmetic. In order to improve the precision of fusion and weed out those underproof data, let mobile robot acquire information from three different directions for every point of location, and build the map on-line according to the step of combination. 3D grid map of belief layout is built based on DSmT shown as Fig 5. Result of recognition of objects in Fig. 4 with DSmT and DST is shown in Fig. 6 and Fig. 7 respectively.

Analysis on the fusion result:

1) In this experiment, 3D grid map of belief layout just based on DSmT is plotted in Fig.5. Seen from it, outline of obstacle and location are expressed clearly, it supplies with a shortcut to do some research on self-localization, path planning and navigation of mobile robot.

2) Though both DSmT and DST can be applied to building grid map from sonar sensors, DSmT can express more clearly the grid information than DST, which can be seen easily from the comparison between Fig.6 and Fig.7 in terms of the grid map of original environment in Fig.4.

3) DSmT can deal with highly conflictive information, while DST can't do. So when we apply DST to grid map building, it might be fail.

4) In this study, though we define two focal elements in either DSm model or D-S one, according to their combination rule, DSmT should have greater amount of computing, however, in fact it is inverse. We analyze, it must take a deal of time to deal with high conflict with DST.

5) Here we give a simple comparison between DSmT and DST from the numbers of acquiring-information spot, the quality of grid map building and the time in need of finishing the global map building in TABLE I. Seen from table 1, DSmT is more superior to DST

\section{CONCLUSION}

In this paper, we applied both DSmT and DST to mobile robot's map building in a small static virtual environment. Through the experiment, DSmT is proved to be more valid than DST finally. Of course, if the size of environment is very

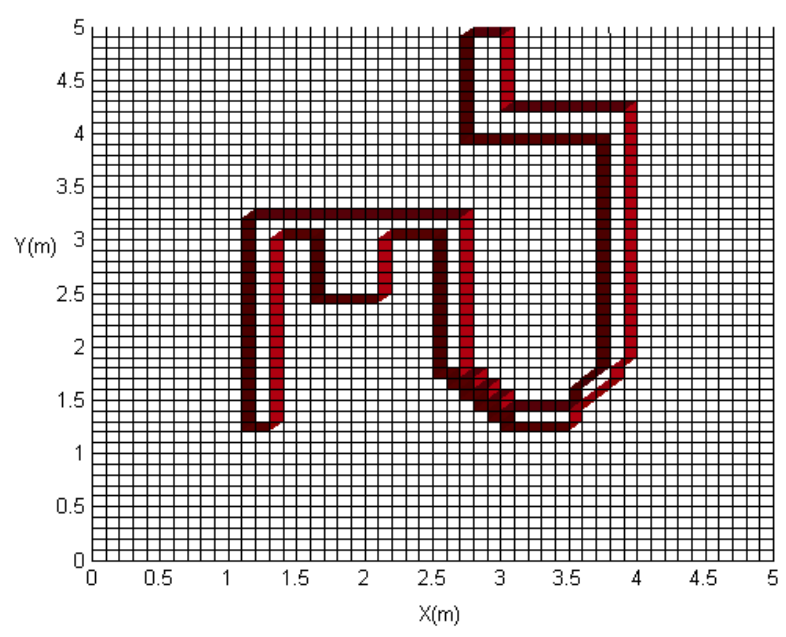

Fig .6: Result of recognition of objects with DSmT

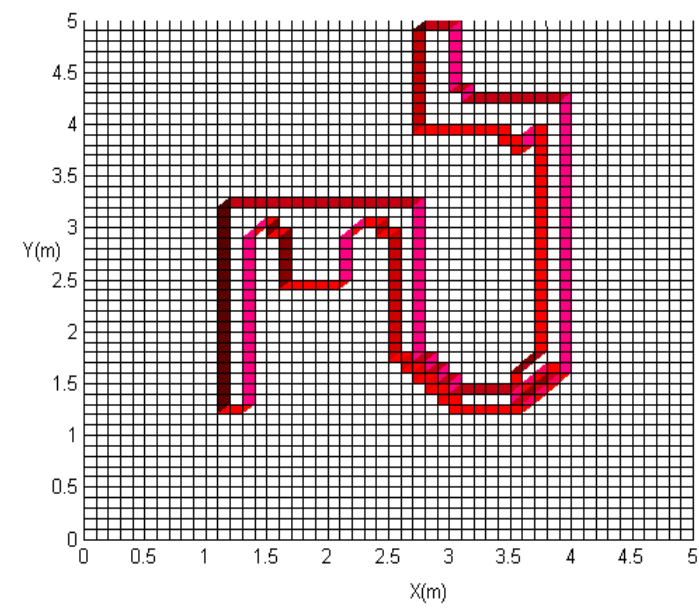

Fig .7: Result of recognition of objects with DST

TABLE I:

Comparison From Grid Map Building Between DSmT and DST

\begin{tabular}{|c|c|c|c|}
\hline Method/Items & $\begin{array}{c}\text { time } \\
(\mathrm{s})\end{array}$ & $\begin{array}{c}\text { Numbers of } \\
\text { spot }\end{array}$ & $\begin{array}{c}\text { Quality of } \\
\text { map }\end{array}$ \\
\hline DST & 356 & 115 & general \\
\hline DSmT & 293 & 97 & high \\
\hline
\end{tabular}

large and complex, or irregular and even loop, and robot's position isn't neglected. We must consider robot's selflocalization. In short, this study supplied with a shortcut for human-computer interface of mobile robot exploring unknown environment and established a firm foundation for us to apply Hybrid DSmT[12] to dynamic unknown environment and multi-robots' building map together.

\section{ACKNOWLEDGMENT}

We are grateful to Dr. Jean Dezert from ONERA/DTIM, Châtillon, France for his guidance, help and technical supports in DSmT.

\section{REFERENCES}

[1]. M. Montemerlo, S. Thrun, "Simultaneous localization and mapping with unknown data association using Fast SLAM, ” IEEE Int Conf. Robotics and Automation, 2003, pp.1985-1991.

[2]. A. J. Dasvison, D. W. Murray, "Simultaneous localization and map building using active vision," IEEE Trans. Pattern Analysis and Machine Intelligence, vol.24, no.7, 2002, pp. 865-880.

[3]. D. Fox, W. Burgard, S.Thrun, "Active markov localization for mobile robots," Robotics and Autonomous Systems, vol.25, no.12, 1998, pp.195-207.

[4]. S. Thrun, D. Fox, W. Burgard, F. Dellaert, "Robust monte carlo localization for mobile robots," Artificial Intelligence, vol. 128, 2001, pp.99-141.

[5]. A. Elfes, H. Moravec, "High resolution maps from wide angle sonar," IEEE Int Conf. Robotics and Automation, 1985, pp.116-121.

[6]. A.Elfes, "Sonar-based real-world mapping and navigation," IEEE J. Robotics Automat, Vol. 3, 1987, pp.249-265.

[7]. G. Shafer, A mathematical theory of evidence, Princeton: Princeton University Press, NJ, 1976.

[8]. P.H. Smets, "Data fusion in the transferable belief model," Proc. of Int Conf. Information Fusion, Paris, July 2000

[9]. J. Dezert, F. Smarandache, "On the generation of hyper-powersets for the DSmT, " Proc. of Int Conf. Information Fusion. pp.1118-1125, Cairns, Queensland, Australia, 8-11 July 2003.

[10]. J. Dezert, F..Smarandache, "Partial ordering of hyper-powersets and matrix representation of belief functions within DSmT," Proc. of Int Conf. Information Fusion, pp.1230-1238, Cairns, Queensland, Australia,8-11 July 2003.

[11]. W.H. Wang, Map building by mobile robots in unknown environment, Ph D, Thesis, Shanghai Jiao Tong University, 2003. 
[12]. F. Smarandache, J. Dezert, Advances and applications of DSmT for information fusion, Rehoboth: American Research Press, 2004. 